\title{
Breast Cancer Awareness
}

\author{
Afa Bayramova* \\ Department of Obstetrics and Gynecology, Yaroslavl State Medical Academy, Russia
}

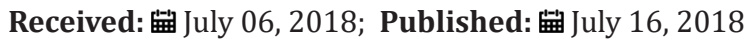

*Corresponding author: Afa Bayramova, Department of Obstetrics and Gynecology, Yaroslavl State Medical Academy, Russia

\begin{abstract}
As an $\mathrm{Ob} \&$ Gyn, member of National Breast Cancer Foundation and a woman I find it very important to raise awareness about the importance of early detection of Breast Cancer. Make a difference! Spread the word about mammograms and encourage communities, organizations, your friends and family members to get involved! Your help matters. Breast cancer is the most common type of tumors among the female population of the United States. One in eight women will be diagnosed with breast cancer in their lifetime.
\end{abstract}

Keywords: Breast cancer; Breast cancer awareness; Gynaecology

\section{Introduction}

Annually, more than one million cases of this disease are registered in the world, and with an average life expectancy of 80 years the risk of developing breast cancer is $12.5 \%$. Over the past 20 years, there has been a steady trend towards an increase in the incidence of this type of cancer, especially in cities and megacities. Since 1985 , he is the first among women with oncological diseases, accounting for $31.2 \%$. It is well known that an annual preventive examination contributes to the early detection of tumors. However, often patients refer to a doctor already with obvious signs of a tumor, and this complicates the subsequent treatment. One of the reasons is the lack of understanding of the importance of early diagnosis of the disease. Now scientists have deeply penetrated into the mechanisms of the origin of the cancer cell, although much is still unclear. Like other cancers, breast cancer remains insidious and aggressive. Therefore, it is very important for any person to have an idea of the development of this disease: it is forewarned, therefore, armed [1].

\section{Why do Women get Breast Cancer?}

There is hardly an unequivocal answer to this quite legitimate question. Breast cancer has been known since time immemorial: its cases are described, for example, in the ancient Egyptian papyri of the doctor Imhotep (3000 BC), in the Babylonian medical code of Hammurabi $(2,250$ years BC); Hippocrates (400 BC) repeatedly refers to it, and Galen (130-200 BC) calls breast tumors cancer because of their external similarity to crab (in Greek the word "crab" and "cancer" sound the same: karkinos). Unlike many diseases, the causes of which are elucidated, the basis for the development of malignant tumors is more complex mechanisms and a variety of causes, including genetic features, environmental factors and many others. Tumors of the breast can be either benign, or malignant, or cancerous. The latter are much less common. Malignant tumors arise accidentally or are associated with heredity. In $10 \%$ of cases, the disease is transmitted from generation to generation and is due to congenital mutations in the BRCA1 or BRCA2 predisposition genes (from English Breast Cancer).

The most common benign disease of the mammary glands is various mastopathy, which refers to a large group of dyshormonal hyperplasia's (proliferation of breast tissue caused by hormonal disorders) and is associated with impaired functions of the ovaries, adrenals, pituitary gland, thyroid gland or with hormone exchange disorders due to liver diseases, obesity, etc. These proliferations of the mammary gland tissue can be in the form of dense nodules, cysts or diffuse formations. Some of these changes can be attributed to precancerous [2]. And that's surprising, breast cancer is found not only in women, but also in men, but much less often: about $1 \%$ of all cases are associated with the male population. Among the causes of mammary tumors in men-a rare mutation in the gene predisposition BRCA2, obesity, hormonal disorders. In contrast to benign growth of breast tissue, malignant degeneration necessarily requires prompt treatment.

It is known that unlike normal cells, tumor cells do not pass after division into a rest phase but continue to grow uncontrollably. Tumor formation is a multistage process. The growth and progression of the tumor, that is, its transition from the initial to 
the more aggressive stage, the tumor growing into the surrounding tissue, or the invasion, depend on the cellular genes. They control the cell cycle, intercellular interactions, aging and cell death, and are also responsible for DNA repair and cell genome stability. Under the influence of various factors in normal genes mutations may occur. The longer cells are divided, the probability of such mutations is higher, which means that the malignant degeneration of cells is more likely due to carcinogens. The accumulation of damage in the cell genome is associated with both DNA replication errors and external, extracellular factors.

To the risk factors at the level of the human body include increased levels of estrogen (female sex hormones), menstrual disorders, thyroid and adrenal gland function, abortions and the lack of breastfeeding. These features are often found in anamnesis of patients with breast cancer. Factors that increase the risk of illness to them include the early age of menarche (11-12 years); late first childbirth (28-30 years); large area and weight of the placenta, increasing the risk of breast cancer in daughters; abortion, especially before the first birth; chronic inflammation of the appendages; endometriosis (proliferation of cells lining the uterus); ovarian cysts and other diseases [3]. At the age after 65 years the second peak of incidence of malignant tumors of the mammary gland associated with adrenal estrogen and increased body weight is observed.

In general, the various risk factors for this disease can be divided into three groups:

a. Related to reproduction (birth of children/abortion, lactation, age of menarche), concomitant gynecological diseases;

b. Related to genetic predisposition: malignant tumors, especially female reproductive organs and mammary glands in close relatives;

c. Associated with metabolic disorders and hormonal disorders, including with the use of hormonal drugs containing estrogens.

\section{Groups and Risk Factors}

A risk factor is any condition or effect that increases the likelihood of the disease. Different types of cancer are associated with various risk factors. It is important to know that the mere fact of having one or even several risk factors for breast cancer does not mean that a woman will automatically fall ill with them. In some women with a number of such factors, the cancer does not develop, whereas in a significant number of patients with breast cancer there are no obvious risk factors. That is why risk factors are divided into groups [4].

\section{High Risk Group}

These are women who have already had cancer of one of the mammary glands. They have an increased risk of developing a new malignant tumor in another breast or in another area of the same breast.

\section{Group of Moderate Risk}

a) Age: The risk of developing breast cancer increases with age. About $77 \%$ of patients with diagnosed breast cancer are over 50 years of age, and half of them are 65 years of age or older.

b) Relationship: Breast cancer in close relatives (mother, sister or daughter) increases the risk of getting sick. The risk is even greater if the cancer of a relative is detected before the onset of her menopause.

c) Genetic factors: The risk is elevated in carriers of altered forms of either of the two genes associated with the "family form" of breast cancer. These are the so-called BRCA1 and BRCA2. In a woman with a hereditary mutation of any of these genes, the risk of developing breast cancer throughout life is close to $80 \%$.

\section{Group of Slightly Increased Risk}

a) Breast cancer in distant relatives (refer to cases of cancer in relatives of not the first degree of kinship of the grandmother, aunts, cousins, etc.).

b) Deviations in the results of previous biopsies. If the results of a biopsy ever indicated the presence of complex component fibroadenomas, hyperplasias, solitary papillomas, then the risk increases insignificantly.

c) Age over 30 years of age at the birth of the first child.

d) Early onset of menstruation. The risk rises if the first menstruation occurred earlier than 12 years.

e) Late menopause. The risk of breast cancer is higher with the onset of menopause after 55 years.

f) Other cancers in the family history. The presence of ovarian, cervical or uterine cancer cases in relatives increases the risk of breast cancer.

g) Prolonged intake of combined estrogen and progesterone drugs increases the risk of developing breast cancer.

\section{Alarming Symptoms}

The development of breast cancer is preceded by the growth of its tissue of hyperplasia (an increase in the number of cells) and the appearance in the ducts of the gland of atypical cells. These changes are called precancerous. These include, and we mentioned mastopathy-cystic fibrotic and diffuse changes in breast tissue. Although malignant degeneration with mastopathy occurs quite rarely, nevertheless the appearance of the first signs of mastopathy, detected by palpation, that is, careful probing of the gland, is an occasion to appeal to a mammologist. Mammography (X-ray examination) and ultrasound are the main methods of early 
detection of breast and mastopathy tumors. The results of these studies are often confirmed by puncture of the mammary gland, when the syringe sample of the alleged neoplasm is examined under a microscope. Very often, the changes in the mammary gland are detected by the patients themselves when examining the glands in the first 6-10 days after the onset of menstruation.

What are the signs that should be alerted to during an independent examination?

\section{Early Signs of Breast Cancer}

Every woman needs regular self-examination. To do this, it is enough to go to the mirror and carefully examine your chest. In most cases, the following symptoms can be seen:

\section{a. Deformation}

b. Swelling

c. An increase or decrease in the breast that has occurred without cause

d. Changes in the nipple or areola zone: erosion, retracted nipple, and discharge from the nipple, which are not associated with pregnancy and feeding

\section{e. Changes on the skin like "lemon crust"}

f. A painless tight formation or a thickened patch in the mammary gland that can be touched by hands.

If you notice one of the above signs, you should immediately call your doctor and be examined. But even if it seems visually that there are no problems, every woman after 40 years of age should regularly, at least once a year, undergo a mammogram [5]. It is also important for women under 40 to be examined by a doctor: annually consult a gynecologist and perform ultrasound of the mammary glands. As studies show, about $80 \%$ of all ill women were able to independently notice the first symptoms of breast cancer. Basically, among all the detected tumoral formations, most of it turns out to be benign. But the help of a specialist - a doctor-mammologist is needed in any case. It is important to remember that the disease, detected at an early stage, is completely curable in almost $90 \%$ of cases, and the third stage can be treated only by $40 \%$.

\section{Without Delay and Panic}

Often, the assumption or conclusion of a doctor-mammologist about the presence of pathological changes in the mammary gland generates the most unpleasant fears in patients, and at times panic. However, to date, there are already many methods to clarify the diagnosis of breast cancer and prescribe appropriate treatment if the diagnosis is confirmed. This is, first of all, the markers (risk factors) of tumor growth found in the blood of patients. Their main purpose is early detection of malignant neoplasms and relapses, as well as evaluation of the effectiveness of treatment of the disease. When referring to a dispensary or a specialized clinic, the patient will be offered to donate blood for the analysis of such markers. With regard to breast cancer, there is not yet a strictly specific marker, so a set of tests for several markers is applied. Concentrations of these markers in the serum of patients depend on the degree of malignancy and stage of the disease and are therefore important for the diagnosis and prognosis of the disease.

There are other parameters that allow a specialist to suspect a disease progression or a metastatic defeat of other organs. Analysis of these indicators, to some extent associated with breast tumors, makes it possible to plan further actions: to prescribe chemo- or hormone therapy, surgical treatment or therapeutic and prophylactic drugs in case of mastopathy or benign tumors, for example, fibroadenoma of the breast. The list of diagnostic tests varies depending on the material capabilities of the clinic and patients, as well as on the specific case of the disease [6]. The main thing is to make timely and professional analyzes, guided by the recommendations of specialists. In life, unfortunately, cases of treatment of patients to various healers and to so-called folk medicine are not uncommon. Without belittling the importance of phytotherapy and homeopathy, oncologists are strongly advised to contact specialized institutions: specialized dispensaries and clinics, where qualified doctors will help the patient. Delay in choosing the right method of therapy is very dangerous.

\section{Complex Treatment}

The basis for the treatment of breast cancer is a combined complex approach combined with surgical treatment. The tumor is affected by drugs, radiation, hormones. Removal of the entire breast, or mastectomy, is often replaced by compromise organpreserving treatment: removal of the glandular segments with axillary lymph nodes. Preserve and restore the shape of the breast can allow reconstructive surgery, the importance of which is obvious, especially for young patients. Operable (subject to surgery) is usually the first-second stage of cancer. With advanced tumors or inoperable cancer, additional treatment is required, which slows the growth of the tumor and allows surgical intervention. However, in most cases, one surgical treatment is not enough. If the risk of recurrence is low, that is, the tumor is small (less than $1 \mathrm{~cm}$ ), there are no metastases to nearby and distant lymph nodes, the degree of malignancy is low, and tumor cells carry the receptors of tumor cells to estrogens and progesterone (the main sex hormones of women), then surgical treatment is often gives a steady positive effect. But sometimes postoperative complications develop in the form of bleeding and wound suppuration. Due to the removal of lymph nodes, lymph drainage from the tissues is broken and lymphostasis develops, usually a few weeks after the operation. Often, lymphostasis is provoked by infections, hot and sunny baths, physical exertion. That is why patients who have undergone a mastectomy are not recommended to inject into the hand on the operated side, there are contra-indicated solariums and saunas, they need to avoid infection of the skin through cracks or scratches. Useful special hand massage and gymnastics, improving the circulation of lymph and restoring the work of joints. 


\section{Success Depends on the Forecast}

The most effective treatment of breast tumors is possible only taking into account the prognosis of the course of the disease and the patient's response to a particular drug, that is, there are a number of factors indicative of a favorable or unfavorable outcome of the disease, as well as the sensitivity of the tumor to the treatment. These factors include tumor size, the presence of lymph node metastases, the tumor histology (its structure determining malignancy), the level of estrogen and progesterone receptors in tumor cells, and others [7].

\section{Morphological and Molecular Biological Factors of Breast Cancer Prognosis and Treatment Effectiveness}

a) Tumor size

b) The number of metastatic lymph nodes

c) Histology of the tumor

d) Germination of the tumor with blood and lymphatic vessels

e) Indicators of DNA synthesis activity

f) Ploidy of tumor cells

g) The intensity of the division of tumor cells (the level of the nuclear protein of mitosin, the level of the nuclear antigen Ki-67, etc.)

h) Presence of estrogen and progesterone receptors

i) The level of activators and inhibitors (blockers) of plasminogen (a protein that participates in many cellular interactions, in invasion and metastasis, etc.).

Many monographs are devoted to the study of these and other factors, we note only those that are of fundamental importance for the development of breast cancer in women. First, it is the receptors of steroid hormones-specific proteins that selectively bind the molecules of the corresponding steroids penetrating into the cells. The presence of receptors in tumor cells for estrogens and progesterone suggests that the tumor is sensitive to hormonal therapy and that there is little possibility of metastasis. The presence of epidermal growth factors in the tumor tissue (substances that promote the growth of certain types of cells) also indicates the sensitivity of the tumor to treatment with hormones. When there are no receptors for steroid hormones in tumor cells, then treating tumors with hormonal therapy is meaningless [8].

The HER2/neu receptor also participates in signaling to cell division. Its blocking can slow or stop the division of tumor cells and the growth of tumors that depend on these signals. For such a blockade, the herceptin preparation was developed. When the gene encoding this receptor is actively transcribed (rewritten), it is advisable to use chemotherapy with the use of herceptin, and hormone treatment in this case is ineffective. Malignant tumors are capable of metastasizing and invading. In these processes, the main role is played by the proteins UPA and PAI-1. If their levels exceed certain minimum values, the risk of breast cancer increases by a factor of 1.5-2, so the data are important for the prognosis. The growth of a malignant tumor is accompanied by the formation in it of a branched network of capillaries-comparatively small blood vessels. The process is regulated by the growth factor of the vascular endothelium. A high level of this protein in the tumor indicates an unfavorable prognosis for both early and progressive malignant processes. Analysis of the activity of the growth factor of the epithelium stimulated the creation of new drugs that block the growth of malignant cells, for example, the promising drug avastin.

Recently it was found out that in the breast tissue with the help of the aromatase enzyme own estrogens are synthesized. Therefore, suppression of the synthesis of this enzyme with drugs, for example arimedex and aromazine, can reduce the risk of breast cancer. Thus, thanks to the research of biochemists and molecular biologists, biologically significant parameters were available to physicians to predict the course of the disease at various stages and to select appropriate therapy for disseminated breast cancer. First, these are the levels of receptors for estrogens and progesterone in the tumor, which make it possible to evaluate the appropriateness of hormone therapy and to foresee its result. Secondly, the level of activity of the Her2/neu gene, allowing to detect patients with an increased risk of recurrence of the breast tumor and the risk of its metastasis in the early stages of the disease. Thirdly, the level of the growth factor of the epithelium, indicating a prognosis of tumor development in the beginning and in the later stages of the disease [9].

\section{Molecular-Biological Background of Breast Cancer}

What molecular mechanisms are at the heart of cancer? It is known that the development of the tumor is associated with a disruption in the growth and differentiation of cells and their malignant degeneration. In many cases, these disorders are caused by mutations or a sharp increase in the activity of cellular oncogenes-normal genes that participate in the regulation of the cell cycle, signaling and other cell life processes. Now we know a little more than 100 different proto-oncogenes-normal cell genes, the increased activity of which changes or a change in their structure leads to the synthesis of oncoproteins, which play a key role in cellular life. Such activation of proto-oncogenes and their transformation into oncogenes are possible as a result of mutations, chromosome rearrangements and other mechanisms [10]. There are about 50,000 functioning genes in the human cell, therefore the share of potential oncogenes responsible for malignant cell growth is quite high. In the case of breast cancer, probably no more than 10 oncogenes are involved.

In addition, cell cycle disorders and uncontrolled multiplication of cells can be associated with mutations in suppressor genes, or tumor suppressors. These include, for example, mutations in the BRCA1 and BRCA2 genes already mentioned. These genes encode 
high molecular weight nuclear proteins that correctDNA damage and maintain the stability of the genome, and the product of the BRCA1 gene participates in the correct distribution of chromosomes in cell division, suppresses excessive hormone-dependent multiplication of breast cells, which occurs, for example, during puberty and lactation pregnant women. That is why the mutations of this gene lead to a whole spectrum of disorders, ranging from new gene mutations and chromosomal rearrangements to the unrestrained reproduction of cells of estrogen-dependent organs that determine the development of breast and ovarian tumors. Thus, mutations in the BRCA1/BRCA2 genes disrupt the control of DNA damage and maintain the integrity of the cellular genome, which causes the appearance of malignant cell clones [11]. In addition to mutations, the activity of tumor suppressor genes and other genes responsible for regulation may change as a result of disorders in the DNA molecule. This is also true of breast cancer [12].

In some laboratories, with the help of special microchips, the profiles of changes in the cells of breast tumors are examined. For example, French researchers compared the profiles of such changes in different samples of malignant cells before and after treatment with doxorubicin, which allowed to outline and substantiate the strategy of chemotherapy. They isolated a group of 496 genes, the activity (expression) of which differs from the expression of these genes in normal mammary tissue. Several years ago, the results of an investigation of the expression of 200 genes, obtained on 122 samples of tumor tissue of the mammary gland, were published. Several groups of breast cancer genes have been isolated, beginning with the expression of genes close to normal and ending with genes with the expression giving the worst prognosis [13]. In many laboratories, experiments are underway to create a "molecular portrait" of breast tumors. Japanese researchers, for example, obtained a molecular profile of genes in the treatment of patients with docetaxel. It turned out that sensitive and resistant to this drug tumors clearly differ in the activity of 76 genes.

Of course, these approaches are still very laborious and expensive to apply in practice, but they are important for the development of more specific and simple methods of analyzing the effectiveness of treatment. Such data will help in the future to predict the course of the disease and the sensitivity of the tumor to therapy. The appearance of cancer can be associated not only with the disruption of the work of oncogenes and other genes, but also with viral infections. In the last 10 years there has been strong evidence that a retrovirus related to the mammary gland tumor virus, or MMTV (from the English Mouse Mammary Tumor Virus) circulates in the human body. Their activation is a complex chain of biochemical processes, similar to the activation of some retroviruses that cause leukemia in humans and animals [14].

First, in the sera of many patients with breast cancer, antibodies to proteins related to the structural proteins of the MMTV membrane were detected. Then, in DNA of peripheral blood lymphocytes and in tumor tissue, in approximately $40 \%$ of patients with sporadic breast cancer, sequences similar in structure to the genes of this retrovirus were detected using highly sensitive methods. From the genome of tumor cells entering the pleural cavity of patients with breast cancer, DNA sequences, 95-97\% corresponding to the fulllength provirus, were isolated and cloned. MMTV-related human provirus, or hMTV (from the English human Mammary Tumor Virus), was detected in the genome of lymphocytes and tumor cells, but not in normal human tissues. This indicates an external source of retroviral infection. The incidence of MMTV-homologous sequences varies from $0-1 \%$ (UK) to $75-80 \%$ (Tunisia). There are several assumptions about the ways of getting the virus into the human body, including infection through the lymphoid tissue of the gastrointestinal tract from contaminated with mouse feces products. The distribution of MMTV-homologous sequences in human populations corresponds to the spread of this species of mice. It is possible to transfer MMTV to the person from the mouse both directly and through intermediate "hosts" (cats, primates).

Recently, a team of researchers from the United States, led by Dr. S. Ross, showed that the primary target for retroviral infection may be tree cells-certain lymphoid cells that absorb foreign proteins and virus particles by pinocytosis-the process of absorbing liquids and foreign bodies. It turned out that these cells can then produce an infectious virus and transmit it to other cells, in particular B-and T-lymphocytes. In the absence of dendritic cells, the effectiveness of MMTV infection decreases dramatically; undoubtedly, it depends on other reasons. For circulation in the body of both mice and humans, MMTV uses peripheral blood lymphocytes. At this stage, the number of copies of provirus in the genome is relatively small. During the active division of the epithelial cells of the mammary gland, growing under the action of hormones, the provirus built into the cellular genome is able to rapidly multiply copies thousands of times. Apparently, the provirus can be built into the cells of the mammary gland, which possess the properties of stem cells, which subsequently give rise to multiple foci of tumor growth. There is evidence of the possible direct or indirect involvement of hMTV in the development of breast cancer. In experiments with cultures of human breast cancer cells containing $(+)$ and not containing $(-)$ proviral sequences, a group of American researchers found that high activity of genes associated with immune response to infection was observed in (+) cells. In addition, in these $(+)$ cells, eight genes that were completely inactive in $(-)$ cells were activated. Comparison of gene expression patterns with microarrays showed that the total activity of genes in infected hMTV cells corresponds to the activity of genes in the inflammatory process involving interferons, that is, a viral infection plays a very definite role in the development of breast cancer [15].

\section{What is the Difference Between Infected and Non- Infected hMTV Tumors?}

Answers to this question were tried by Australian biologists. They showed that in the early stages of breast cancer in patients of one of the Sydney clinics infected with this retrovirus, the tumors 
in their structure and growth are similar to mammary tumors in mice. However, some similarities in the development of mammary and human breast cancer were noted long before this work. Both in humans and in mice, breast tumors are known, in which very rapid growth begins immediately in several foci. There is reason to believe that such tumors arise from stem-type cells that have acquired the ability to malignant growth due to either genomic disorders in cells or a viral infection. New in the study of Australian scientists was the fact of accumulation in the nuclei of infected hMTV human breast tumors of inactive protein-the product of the p53 gene suppressing tumor growth, which we mentioned above. Why this protein does not work, it remains to be seen, but such tumors are characterized by faster growth, most often they are localized in the area of the ducts of the mammary glands [15].

At the same time, a group of researchers led by Profsohmer Selmont from Austria, using genetic engineering structures, showed that the product of one of the MMTV genes contains an amino acid sequence corresponding to amino acid sequences in the immunoreceptors, or ITAM from Immunoreceptor Tyrosinebased Activation Motifs, involved in the transmission of signals that regulate the development of cells. The hyperactivation of these signaling pathways due to the manifestation of provirus in epithelial cells of the breast can lead to cell degeneration and the development of breast cancer. The possibility of the appearance of morphologically altered cells capable of three-dimensional growth in a culture medium in vitro was tested by us in experiments with human embryonic kidney epithelial cells that were cultured with virus-producing mouse cells. It has been shown that viral sequences are indeed embedded in the cellular genome and RNA transcripts are formed on them. The introduction of provirus into the genome of a cell can lead to a change in the activity of genes in the area of such a combination. In addition, in the mouse retroviral genome, there are hormone-sensitive regulatory elements that can also alter or completely disrupt the functioning of cellular genes. Let us also recall the encoded MMTV amino acid sequences in ITAM that can mediate significant changes in virus-infected cells. However, in subsequent cell division as a result of chromosome rearrangements, some of the chromosomes are lost along with the copies of the provirus embedded in the cellular gene. Thus, tumor degeneration is likely only in a limited number of cells.

In other words, in addition to the horizontal transmission of hMTV, there seems to be a vertical one: from mother to child. In this case, lymphoid tissue of the human intestine can serve as a "gateway" of retroviral infection, and lymph nodes - a reservoir. It is important that when multiplying the number of proviral copies in the cell genome, a minimal immune response to infection is observed, although signs of inflammation are found in infected cells. For example, as already mentioned, genes encoding proteins that are involved in regulatory processes with interferon are active. According to some researchers, the inflammation in the mammary glands, as well as their sharp involution, that is, mass reduction of tissues (for example, after abortion or sudden cessation of lactation), may increase the risk of malignant cell degeneration. The involvement of MMTV in the development of a malignant tumor, and possibly also tumors of other localizations, allows isolating carriers of hMTV to a risk group along with patients from hereditarily burdened families carrying mutations in the genes of predisposition [16].

So, thanks to the efforts of specialists in the field of clinical biochemistry and genetics, molecular biology, virology and immunology, it was possible to compile a fairly capacious "molecular portrait" of breast cancer. While the efforts of scientists are focused on elucidating the causes and mechanisms of the onset of tumors, doctors seek to recognize tumors at possibly early stages that are well amenable to treatment and prognosis. That is why the number of parameters for cancer diagnosis and evaluation of its development continues to grow. About some of them we told in the article offered to the reader. Which of these markers will be most effective and will find application in the clinic, will show the near future [17]. Although inexorable statistics indicate an increase in the incidence of breast cancer, experts have reason for optimism. As Dr. Selmon pointed out: "As far as cancer is concerned, the human mind is so ingenious." Undoubtedly, early preclinical diagnostics can now significantly improve the results of treatment and achieve better survival of patients, return them to active life and give hope for a full recovery.

\section{Factors of Heredity}

Breast cancer in $10 \%$ of cases is inherited. If one of the two copies or alleles of the BRCA1 gene in a woman carries a mutation, the probability of developing breast cancer increases to $85 \%$, and the cancer of the ovaries-up to $40 \%$. In carriers of such mutations, the risk of colon cancer increases, and in men-prostate cancer. In the BRCA1 gene, up to 700 different mutations are detected, which are typical for women in certain geographical regions. In the case of mutations in the BRCA2 gene, the risk of developing malignant tumors of the breast and ovaries is relatively lower. It is shown that the frequency of mutations in the BRCA1 gene in patients with familial breast cancer is about an order of magnitude higher than in the BRCA2 gene. Mutations in the BRCA1 gene are found in $16 \%$ of families with two or more close relatives suffering from malignant breast tumors in the absence of ovarian cancer. The combination of these two diseases in the family is also due to mutations in the BRCA1/BRCA2 genes. It turned out that mutations in the BRCA1 gene are found in $61 \%$ of patients with family cases of breast and ovarian cancer. Mutations in the BRCA1/BRCA2 genes can cause other forms of cancer.

Carriers of these mutations need a regular visit to the mammologist, since they have a risk of breast cancer reaching $80-90 \%$, and their nearest relatives are advised by a geneticist. In addition to these, there are other genes, mutations or certain allelic variants of which can promote the development of breast tumors 
and some other malignant tumors. However, this contribution is not as significant as in the case of the BRCA1/BRCA2 genes. The hypothesis of a possible mutation arises in the geneticist if two or more breast cancer patients are found among the immediate relatives of the patient, if the disease develops before the age of 45 , with tumors in two mammary glands or in cancer with multiple foci of the tumor, and in the presence of cancer ovaries. Sporadic breast cancer develops for no apparent reason, although there are certain factors, not related to hereditary predisposition, increasing the risk of its occurrence.

\section{References}

1. National Breast Cancer Foundation.

2. Sorlie T, Perou CM, Tibshirani R, Aas T, Geisler S, et al. (2001) Gene expression patterns of breast carcinomas distinguish tumor subclasses with clinical implications. Proc Natl Acad Sci U S A 98(19): 10869-10874.

3. McAllister SS, Weinberg RA (2014) The tumour-induced systemic environment as a critical regulator of cancer progression and metastasis. Nat Cell Biol 16(8): 717-727.

4. Kitamura T, Qian BZ, Pollard JW (2015) Immune cell promotion of metastasis. Nat Rev Immunol 15(2): 73-86.

5. Lin EY, Li JF, Gnatovskiy L, Deng Y, Zhu L, et al. (2006) Macrophages regulate the angiogenic switch in a mouse model of breast cancer. Cancer Res 66(23): 11238-11246.

6. Noy R, Pollard JW (2014) Tumor-associated macrophages: from mechanisms to therapy. Immunity 41(1): 49-61.

7. Franklin RA, Liao W, Sarkar A, Kim MV, Bivona MR, et al. (2014) The cellular and molecular origin of tumor-associated macrophages. Science 344(6186): 921-925.
8. Campbell MJ, Wolf D, Mukhtar RA, Tandon V, Yau C, et al. (2013) The prognostic implications of macrophages expressing proliferating cell nuclear antigen in breast cancer depend on immune context. PLoS One 8(10): 79114.

9. Mukhtar RA, Moore AP, Tandon VJ, Nseyo 0, Twomey P, et al. (2012) Elevated levels of proliferating and recently migrated tumor-associated macrophages confer increased aggressiveness and worse outcomes in breast cancer. Ann Surg Oncol 19(12): 3979-3986.

10.Wynn TA, Chawla A, Pollard JW (2013) Macrophage biology in development, homeostasis and disease. Nature 496(7446): 445-455.

11. Joyce JA, Pollard JW (2009) Microenvironmental regulation of metastasis. Nat Rev Cancer 9(4): 239-252.

12.Qian BZ, Pollard JW (2010) Macrophage diversity enhances tumor progression and metastasis. Cell 141(1): 39-51.

13. Pollard JW (2004) Tumour-educated macrophages promote tumour progression and metastasis. Nat Rev Cancer 4(1): 71-78.

14. Condeelis J, Pollard JW (2006) Macrophages: obligate partners for tumor cell migration, invasion, and metastasis. Cell 124(2): 263-266.

15. Bingle L, Brown NJ, Lewis CE (2002) The role of tumour-associated macrophages in tumour progression: implications for new anticancer therapies. J Pathol 196(3): 254-265.

16. Perk J, Iavarone A, Benezra R (2005) Id family of helix-loop-helix proteins in cancer. Nat Rev Cancer. 5(8): 603-614.

17. Badve S, Dabbs DJ, Schnitt SJ, Baehner FL, Decker T, et al. (2011) Basal-like and triple-negative breast cancers: a critical review with an emphasis on the implications for pathologists and oncologists. Mod Pathol 24(2): 157-167.

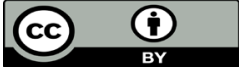

This work is licensed under Creative Commons Attribution 4.0 License

Submission Link: Submit Article

DOI: 10.32474/OAJRSD.2018.01.000111

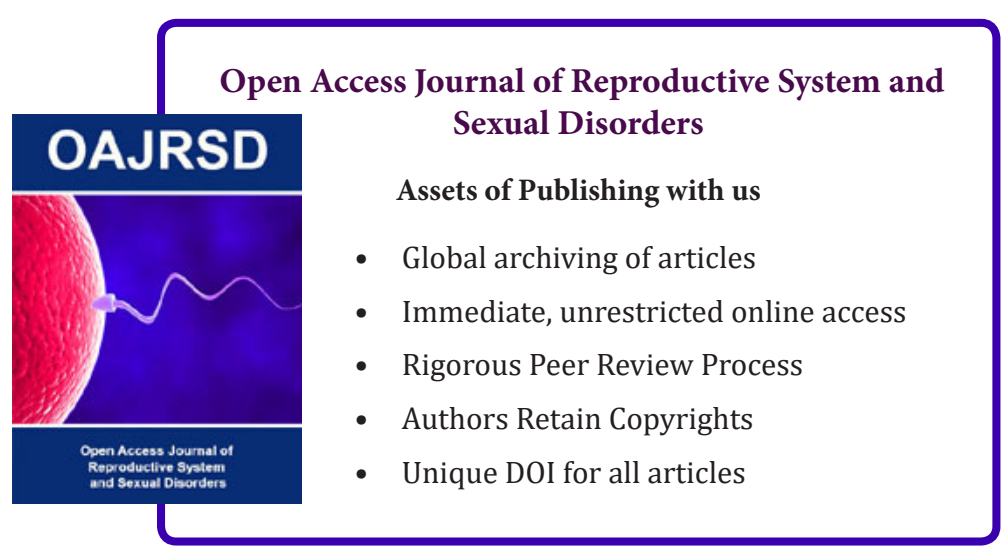

